EPJ manuscript No.

(will be inserted by the editor)

\title{
TMDs and GPDs at a future Electron-Ion Collider
}

\author{
Rolf Ent ${ }^{1 \mathrm{a}}$ \\ Jefferson Lab, 12000 Jefferson Avenue, Newport News, VA 23606 \\ Received: date / Revised version: date
}

\begin{abstract}
In the U.S., an Electron-Ion Collider (EIC) of energy $\sqrt{s}=20-100 \mathrm{GeV}$ is under design, with two options studied at Brookhaven National Lab and Jefferson Laboratory. The recent 2015 US Nuclear Science Long-Range Planning effort included a future EIC as a recommendation for future construction. The EIC will be unique in colliding polarised electrons off polarised protons and light nuclei, providing the spin degrees of freedom essential to pursue its physics program driven by spin structure, multi-dimensional tomographic images of protons and nuclei, and discovery of the role of collective effects of gluons in nuclei. The foreseen luminosity of the EIC, coupled with its energy variability and reach, will allow unprecedented three-dimensional imaging of the gluon and sea quark distributions, via both TMDs and GPDs, and to explore correlations amongst them. Its hermetic detection capability of correlated fragments promises to similar allow for precise tomographic images of the quark-gluon landscape in nuclei, transcending from light few-body nuclei to the heaviest nuclei, and could uncover how the TMD and GPD landscape changes when gluons display an anticipated collective behavior at the higher energies.
\end{abstract}

PACS. PACS-key discribing text of that key - PACS-key discribing text of that key

\section{Introduction}

Our view of the structure of the atomic nuclei and the nucleons they contain has made quite a transformation in the last decades. A common picture found in textbooks shows a 3-valence quark structure of the nucleon, yet we now know that the inside of the nucleon is rather a complex many-body system with a large number of gluons and sea quarks. There is unambiguous evidence that these both play unexpected roles for defining the structure of nuclear matter around us. In order to understand how the properties and structure of all forms of nuclear matter emerge from the dynamics encoded in QCD, it is essential to precisely image gluons and sea quarks, and to understand the role they and their interactions play in protons, neutrons, and nuclei $[1,2]$.

For this, a new accelerator facility is required - the Electron-Ion Collider (EIC). EIC must make a qualitative leap in technical capabilities beyond previous electron scattering programs. It must reach collision energies far higher than are available even at the upgraded CEBAF. It will exceed the earlier $e p$ collider HERA by providing:

- Luminosity a factor of 100-1000 times higher, allowing unprecedented three-dimensional imaging of the gluon and sea quark distributions and to explore correlations among them

a The author wishes to acknowledge the efforts of the global Electron-Ion Collider family, to whom much of the material and ideas are owed, and especially Abhay Deshpande, Zein-Eddine Meziani and Jianwei Qiu, for ably editing the EIC white paper.
- Extensive energy variability to explore the transition in nuclear properties from the region of sea quarks to that of abundant gluons at low $x$, down to 0.001 or 0.0001 or so

- Spin-polarised proton and light ion beams to explore the correlations of gluon and sea quark distributions with the overall nucleon spin, and the contribution of gluons and sea quarks to the nucleon-nucleon interaction

- Heavy-ion beams to reach much higher gluon densities than with proton beams, to study the role and behaviour of gluons in nuclei, and to enhance the discovery of collective effects of gluons

\section{EIC Machine Configuration}

The suite of worldwide facilities, current or anticipated to be in operation, does not provide the capabilities required to complete our understanding of the QCD structure of nuclear matter in all its forms. Much evidence points to the missing link being a polarised, high luminosity EIC, where physicists can probe both nucleons and nuclei in a regime where their properties are dominated by sea quarks and abundant gluons.

Two designs for a future EIC have evolved in the United States. At Brookhaven National Laboratory (BNL) the eRHIC design (Fig. 1, left) utilizes a new electron beam facility based on an Energy Recovery LINAC (ERL) to be built inside the RHIC tunnel to collide with existing highenergy polarised proton and nuclear beams [3]. At Jeffer- 
son Laboratory (JLab) the JLEIC design (Fig. 1, right) employs a new electron and ion collider ring complex together with the $12 \mathrm{GeV}$ upgraded CEBAF to achieve similar collision parameters $[4,5]$.

The EIC machine designs are aimed at achieving

- Highly polarized ( $70 \%$ ) beams of electrons, protons and light nuclei

- Ion beams from deuteron to the heaviest nuclei (uranium or lead)

- Variable $\sqrt{s}$ from $\sim 20$ to $\sim 100 \mathrm{GeV}$, upgradable to $\sim 140 \mathrm{GeV}$

- High collision luminosity $\sim 10^{33-34} \mathrm{~cm}^{-2} \mathrm{~s}^{-1}$

- Possibility to have more than one interaction region

The EIC requirements will push accelerator designs to the limits of current technology to be validated by, partly ongoing, research and design. Cooling of the hadron beam is essential to attain the luminosities demanded by the science. The development of coherent electron cooling is underway at BNL, while the JLab design is based on conventional electron cooling techniques, but proposes to extend them to higher energy and to use bunched electron beams for the first time. An ERL at the highest possible energy and intensity is key to the realisation of eRHIC at BNL, and this technology is also important for electron cooling in JLEIC at JLab. The eRHIC design at BNL requires a high intensity polarised electron source that would be an order of magnitude higher in intensity than the current state of the art, while the JLEIC design at JLab will utilize a novel figure- 8 storage ring design for both electrons and ions.

The physics-driven requirements on the EIC accelerator parameters combined with the demands of full kinematic coverage for measurements make the integration of the detector into the accelerator a particularly challenging feature of the design. Lessons learned from past experience at HERA strongly influenced the design of the EIC interaction region. Driven by needs for high precision on particle detection and identification of final state particles in both $e p$ and $e A$ programs, modern particle detectors will be at the heart of the EIC.

\section{The 3D Structure of Matter}

Several decades of experiments on deep inelastic scattering (DIS) of electron or muon beams off nucleons have taught us about how quarks and gluons (collectively called partons) share the momentum of a fast-moving nucleon. They have not, however, resolved the question of how partons share the nucleon's spin and build up other nucleon intrinsic properties, such as its mass and magnetic moment. The earlier studies were limited to providing the longitudinal momentum distribution of quarks and gluons, a one-dimensional view of nucleon structure. The EIC is designed to yield much greater insight into the nucleon structure by facilitating multi-dimensional maps of the distributions of partons in space, momentum (including momentum components transverse to the nucleon momentum), spin, and flavor. In such studies one must go beyond DIS in that either one or more hadrons produced in the electron-quark scattering process are detected, socalled semi-inclusive deep inelastic scattering (SIDIS) experiments, or the complete final state is detected in socalled deep exclusive scattering experiments. Measurements of both of these processes enable the multi-dimensional mapping of the partons, requiring high luminosity for sufficient statistical precision to be achieved in the multidimensional kinematics governing these studies.

The $12 \mathrm{GeV}$ upgrade of CEBAF at JLab [6] and COMPASS at CERN [7] will accomplish such studies, predominantly in the valence quark region and somewhat extending into the sea quark region. However, these programs will be dramatically extended at the EIC to explore the role of the gluons and sea quarks in determining the hadron structure and properties.

Explicitly, the EIC will enable measurements of:

- the distribution of sea quarks and gluons in momentum and in position space,

- their polarization and their orbital angular momentum, the latter being closely connected with their transverse position and transverse motion since it is a cross product $(\boldsymbol{L}=\boldsymbol{r} \times \boldsymbol{p})$,

- correlations between the polarization and distribution of partons in momentum or position space, which may be regarded as the QCD analog of spin-orbit correlations in atomic or nuclear physics.

Especially, the EIC will acquire unique information on the changes of distributions when going from small $x$ (a few $\times 0.0001)$ to large $x$ (a few $\times 0.1)$, to compare the characteristics of gluons, sea and valence quarks, in order to understand their relation and dynamical interplay. Likewise, it will allow the dependence of the above characteristics on the quark flavor. This is of particular interest when comparing light-sea quark flavor distributions, i.e., $\bar{u}$ with $\bar{d}, \bar{s}$ with $(\bar{u}+\bar{d}) / 2$, or $s$ with $\bar{s}$. Significant differences between those distributions are a direct imprint of non-perturbative dynamics because perturbative parton radiation is not able to generate them. This imparts special interest to the polarization carried by sea quarks of different flavors, above and beyond their contribution to the overall spin of the proton, even if small.

\subsection{The Confined Motion of Partons Inside the Nucleon}

SIDIS measurements have two natural momentum scales: the large momentum transfer from the electron needed to achieve the desired spatial resolution, and the momentum of the produced hadrons perpendicular (or transverse) to the direction of the momentum transfer, which prefers a small value sensitive to the motion of confined partons. Remarkable theoretical advances over the past decade have led to a rigorous framework where information on the confined motion of the partons inside a fast moving nucleon is matched to transverse momentum dependent parton distributions (TMDs). In particular, TMDs 

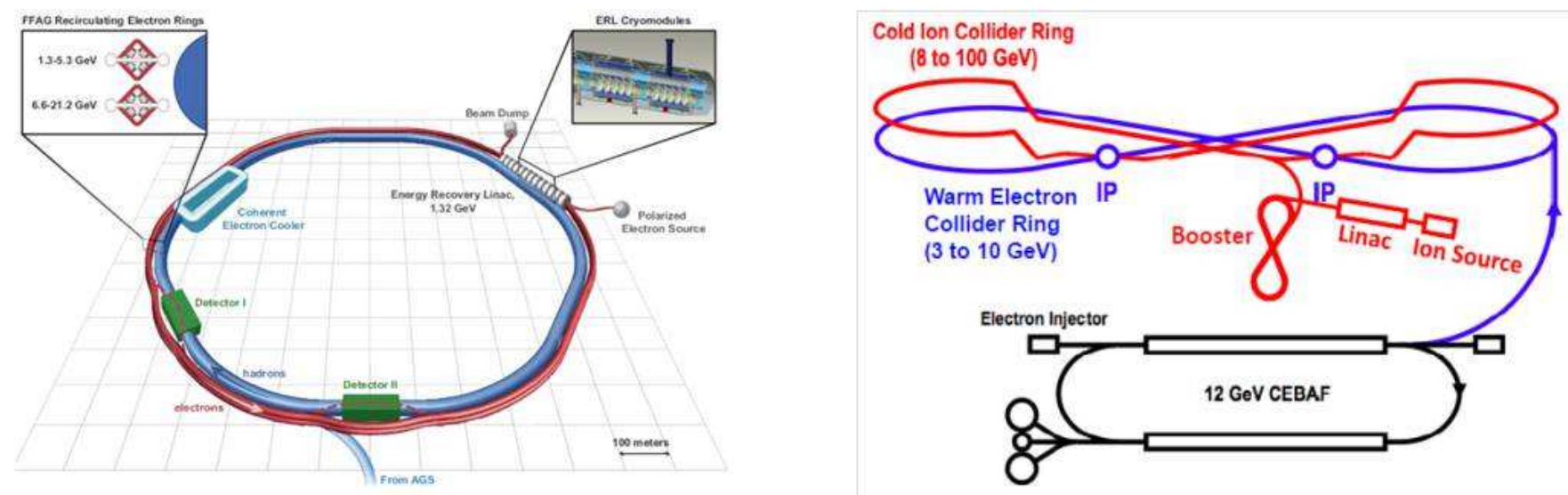

Fig. 1. Left: The schematic of eRHIC at BNL, which would require construction of an electron beam facility to collide with one of the RHIC beams at up to three interaction points. Right: The schematic layout of JLEIC at JLab, which would require construction of an ion linac and ion collider ring, and an electron collider ring with at least two interaction points, around the $12 \mathrm{GeV}$ CEBAF.

are sensitive to correlations between the motion of partons and their spin, as well as the spin of the parent nucleon. These correlations can arise from spin-orbit coupling among the partons, about which very little is known to date. TMDs thus allow us to investigate the full threedimensional dynamics of the proton, going well beyond the information about longitudinal momentum contained in conventional parton distributions. With both electron and nucleon beams polarised at collider energies, the EIC will dramatically advance our knowledge of the motion of confined gluons and sea quarks in ways not achievable at any existing or proposed facility.

The power and rich possibilities of the TMD approach arise from the simple fact that the parton's transverse momentum is a vector $k_{T}$, which allows for various correlations with the other vectors involved: the nucleon momentum $P$, the nucleon spin $S$, and the parton spin. Accordingly, there are eight independent TMD quark distributions. The "simplest" of these is the unpolarized function $f_{1}^{q}\left(x, k_{T}\right)$ which describes, in a fast moving nucleon, the probability of finding a quark carrying the longitudinal momentum fraction $x$ of the nucleon momentum, and a transverse momentum $k_{T}$. Recently, there has been progress in a possible (up and down) flavor separation of this information [8]. Because of the correlations between the quark transverse momentum and the nucleon (or quark) spin, the TMDs naturally provide important information on the dynamics of partons in the transverse plane in momentum space, as compared to the GPDs below which describe the dynamics of partons in the transverse plane in position space. Measurements of the quark TMDs provide then information about the correlation between the quark orbital angular momentum and the nucleon (or quark) spin, essentially as they retain some sensitivity to the cross product $\boldsymbol{r} \times \boldsymbol{p}$. This can then provide important information to disentangle the quark orbital angular momentum.

A prominent TMD example, closely intertwined with the process of dynamical chiral symmetry breaking, is the quark Sivers function $f_{1}^{q}\left(x, k_{t}, S_{T}\right)$ which describes the transverse momentum distribution of unpolarised quarks correlated with the transverse polarization vector of the nucleon. As a result, the quark distribution will be azimuthally asymmetric in the transverse momentum space. Fig. 2 demonstrates the deformations of the up (left) and down (right) quark distributions with a model calculation consistent with current experimental HERMES, COMPASS and JLab data [9]. The center panel of Fig. 2 illustrates the achievable statistical precision of the quark Sivers function from EIC kinematics, ranging from $x \sim$ 0.001 to a few $\times 0.1$. Of course, this represents an extrapolation as nothing is known to date on the spin and momentum correlations of the gluons and sea quarks, as currently no data exist for extracting such pictures in the gluon-dominated region of the proton. Yet, the achievable quality of EIC projected data is evident.

Just as there are eight TMDs for quarks, there exist eight TMDs for gluons. Experimentally, the gluon TMDs and in particular the gluon Sivers function are completely unexplored so far and existing facilities wil likely not probe them. At an EIC, however, many processes could be used to probe the transverse momentum dependent gluon distributions. One example is electroproduction of a heavy open-charm meson pair $(D \bar{D}), \gamma^{*} N\left(S_{T}\right) \rightarrow D\left(k_{1}\right)+\bar{D}\left(k_{2}\right)+$ $X$, where $N\left(S_{T}\right)$ represents a transversely polarized nucleon, and $D$ and $\bar{D}$ are the two mesons with momenta $k_{1}$ and $k_{2}$, respectively. Similar to the Sivers effect in semiinclusive hadron production of above, the gluon Sivers function will introduce an azimuthal asymmetry correlating the total transverse momentum $k_{T}$ of the $(D \bar{D})$ pair with the transverse polarization vector $S_{T}$ of the nucleon. This will result in a single-spin azimuthal asymmetry. It has been shown that such measurements are feasible at an EIC and in principle have sensitivity to the gluon Sivers function. This would constitute a first measurement of such a gluon (TMD) Sivers effect. [2] 

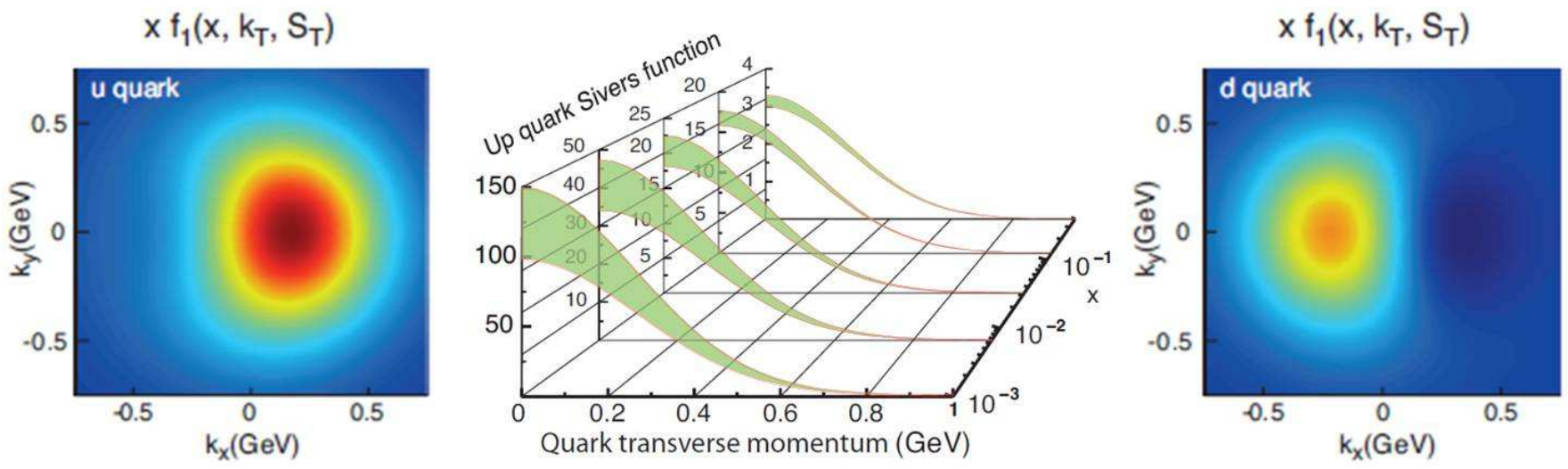

Fig. 2. The density in the transverse-momentum plane for unpolarized up quarks (left) and down quarks (right) with longitudinal momentum fraction $x=0.1$ in a transversely polarized proton moving in the $z$-direction, while polarized in the $y$-direction. The anisotropy due to the proton polarization is described by a model for the Sivers function [9]. The color code indicates the probability of finding the up quarks. The deep red (blue) indicates large negative (positive) values for the Sivers function. Center: The transverse-momentum profile of the up quark Sivers function at five values of $x$ as accessible to the EIC, and corresponding statistical uncertainties.

\subsection{Nucleon Tomography - Spatial Imaging of Gluons and Sea Quarks}

By choosing particular final states in $e p$ scattering, the EIC will probe the transverse spatial distribution of sea quarks and gluons in the fast-moving proton as a function of the parton's longitudinal momentum fraction, $x$ (see an illustration in Fig. 3, left). This spatial distribution yields a picture of the proton that is complementary to the one obtained from the transverse-momentum distribution of quarks and gluons, revealing aspects of proton structure that are intimately connected with the dynamics of QCD at large distances - where the coupling of QCD is strong.

The tomographic images obtained from cross-sections and polarization asymmetries for deep exclusive processes are encoded in generalised parton distributions (GPDs) that unify the concepts of parton densities and of elastic form factors. They contain detailed information about spin-orbit correlations and the angular momentum - their spin and their orbital motion - carried by partons. The combined kinematic coverage of the EIC and of the upgraded CEBAF as well as COMPASS is essential for extracting quark and gluon angular momentum contributions to the proton's spin.

With its broad range of collision energies, its high luminosity and nearly hermetic detectors, the EIC could image the proton with unprecedented detail and precision from small to large transverse distances. The accessible parton momentum fractions $x$ extend from a region dominated by sea quarks and gluons to one where valence quarks become important, allowing a connection to the precise images expected from the $12 \mathrm{GeV}$ upgrade at JLab and COMPASS at CERN. The kinematic access the EIC provides for a range in $x$ transitioning from the region of valence quarks, roughly above $x \sim 0.1$, through the region where the sea quarks may contribute to non-perturbative nucleon structure, roughly $0.01<x \leq 0.3$, into the region where gluons are abundant, roughly down to $x=0.0001$ or 0.001 . To have sufficient resolution, hermeticity and luminosity to measure deep exclusive reactions over this range has been one of the fundamental assumptions of the EIC design.

Deeply virtual Compton scattering (DVCS) is measured in the reaction $e p \rightarrow e p \gamma$ and plays a privileged role in several respects: (i) its theoretical understanding is most advanced; (ii) it has a large number of angular and polarization observables that can constrain GPDs; (iii) Compton scattering interferes with the Bethe-Heitler process (where the incident or scattered electron emits the photon, rather than the electron-quark scattering process), which allows one to extract the complex phase of the Compton scattering amplitude, in turn further constraining the GPDs. Thus, Compton scattering has the potential to yield detailed and precise information about GPDs for different polarizations of the partons and the proton.

DVCS measurements for imaging partons with $x$ between 0.1 and 0.01 , in the transition region between valence and sea quarks, will be possible with the COMPASS experiment at CERN, which will have the benefit of both $\mu^{+}$and $\mu^{-}$beams to also measure the charge asymmetries. The anticipated integrated luminosity promises measurements up to $Q^{2}=10 \mathrm{GeV}^{2}$ or so, but will, however, limit the accuracy of measurements at $Q^{2}$ above, and constrain the possibilities to explore simultaneously the multi-dimensional dependence on $x, Q^{2}$ and $t$. A first era of precise parton imaging with DVCS measurements will begin with the $12 \mathrm{GeV}$ upgrade at JLab, with very high statistics and sufficiently high $Q^{2}$ to probe partons at high- $x(>0.1)$, including the effects of polarization.

A limitation of DVCS is that it is sensitive only to the sum of quark and anti-quark distributions in a particular flavor combination, just as the inclusive DIS process, and that it involves gluon distributions only via a logarithmic dependence on $Q^{2}$. Similar to the case of inclusive DIS, where SIDIS is needed to provide sensitivity towards the different quark flavors, exclusive meson production can offer substantial help to Compton scattering in the separation of different quark and antiquark flavors and even 

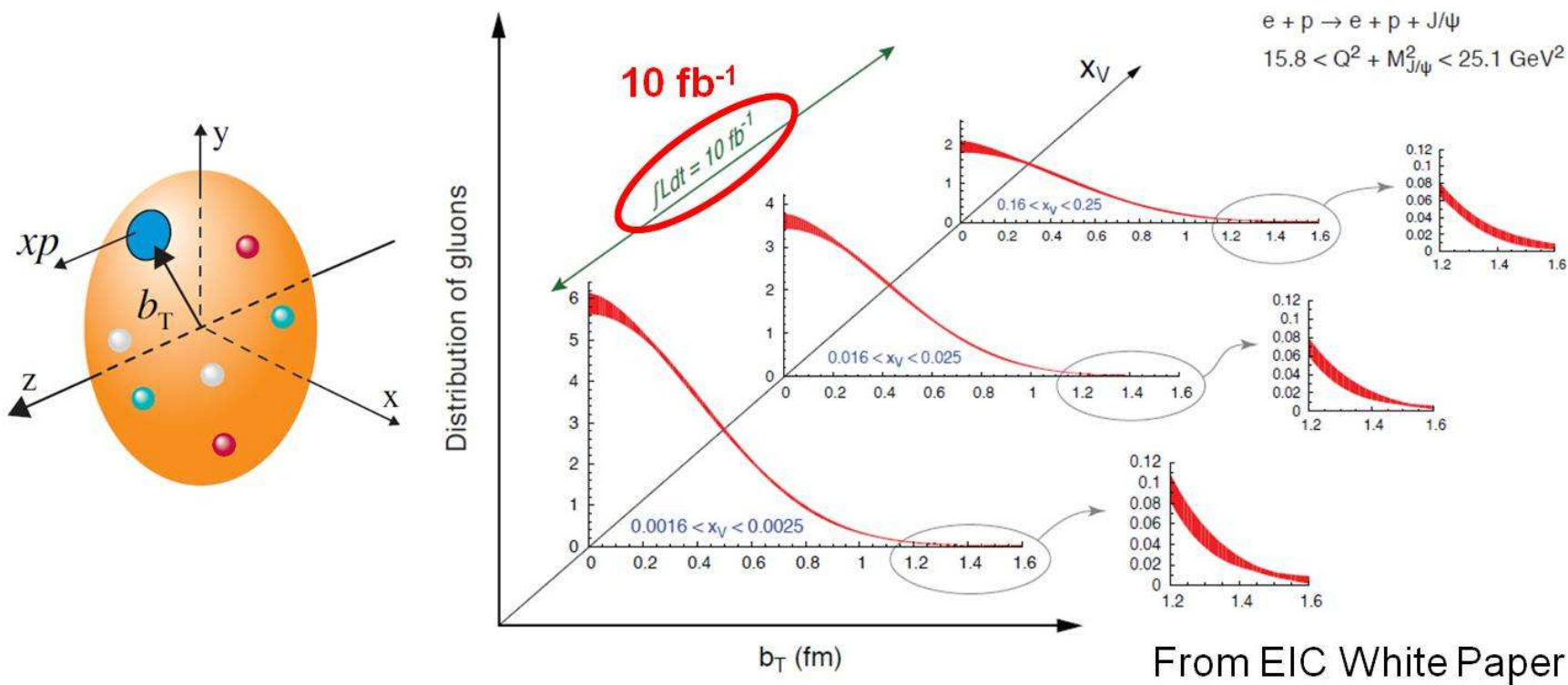

Fig. 3. Left: Schematic view of a parton with longitudinal momentum fraction $x$ and transverse position $b_{T}$ in the proton. Right: The projected precision of the transverse spatial distribution of gluons as obtained from the cross-sections of exclusive $J / \Psi$ production at an EIC [2]. The distance of the gluon from the center of the proton is $b_{T}$ in femtometers, and the kinematic quantity $x_{V}=x_{B}\left(1+M_{J / \Psi}^{2} / Q^{2}\right)$ determines the gluon's momentum fraction.

of gluons. The theoretical description of these exclusive meson production processes is however more involved and theoretical progress is still needed. Measuring at $Q^{2}$ well above $10 \mathrm{GeV}^{2}$ is thought to substantially decrease the theoretical uncertainties. This holds in particular for parton imaging, given that at lower $Q^{2}$ the measured $t$ dependence receives contributions from the finite meson size as well as from the structure of the proton target under study.

The EIC will be unmatched to measure these processes over a large range in $x$ and $Q^{2}>10 \mathrm{GeV}^{2}$. For example, the detection of exclusive $J / \Psi$ meson production would provide unprecedented maps showing how the gluons are distributed in space within a plane perpendicular to the parent proton motion (see Fig. 3, right), as defined within the GPD formalism above. Such particular maps encode vital information, inaccessible without EIC, on the amount of proton spin associated with the gluons' orbital motion through the correlation of a longitudinal momentum component $x$ and a transverse spatial position $b_{T}$.

\section{A Spin Facility}

Spin is an essential property of any particle, and parton distributions can depend on the polarization of both the parton and the parent proton. The spin structure is particularly rich for the TMDs and GPDs discussed above because they single out a direction in the transverse plane, thus opening the way for studying correlations between spin and transverse momentum $k_{T}$ or transverse spatial position $b_{T}$. Information about these transverse degrees of freedom is essential to access orbital angular momentum. But to complete the picture of what dynamically constitutes the spin of the proton, precise and unambiguous maps of the helicity distributions of the sea quarks and gluons are equally required.

An intense, worldwide experimental program over the past decades has shown that the spin of quarks and antiquarks is only responsible for $\sim 30 \%$ of the proton spin. Recent RHIC results indicate that the gluons' spin contribution in the currently explored kinematic region is nonzero, but not yet sufficient to account for the missing $70 \%$ of the proton's spin. The EIC due to its emphasis on polarised electrons colliding with polarised protons and light ions, will settle this conundrum.

The energy range of the EIC gives access to the region of small- $x$ of relevance to the DIS experiments. The partons' total helicity contribution to the proton spin is known to be sensitive to the minimum $x$ accessible by the existing experiments. With the unique capability to reach two orders of magnitude lower in $x$ and to span a wider range of momentum transfer $Q$ than previously achieved, the EIC would offer the most powerful tool to precisely quantify how the spin of gluons (see Fig. 4, right) and that of quarks of various flavors contribute to the proton's spin. The EIC would realize this by colliding longitudinally polarised electrons and nucleons, with both inclusive and semi-inclusive DIS measurements.

EIC will make a huge impact on our knowledge of the gluon's helicity contribution $\Delta(\mathrm{G})$, reducing the present uncertainties by roughly a factor of 10 , combined with a $\sim 2-3$ impact on the quark helicity contribution $\Delta(\Sigma)$. Such gains in knowledge will be unmatched by any other existing or anticipated facility. This would definitively resolve any question of whether parton spin preferences alone can account for the overall proton spin, or whether addi- 

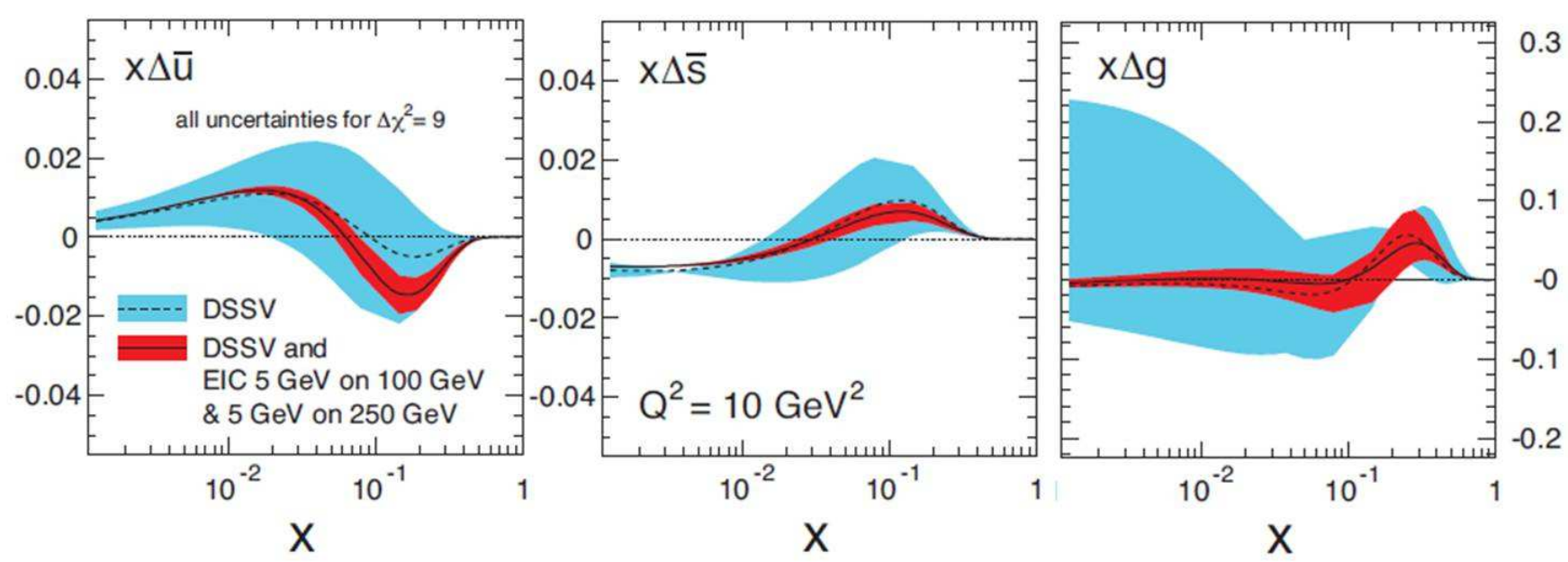

Fig. 4. Left: Uncertainty bands on the $\Delta \bar{u}$ helicity parton distribution in an analysis by DSSV [10] without (light bands) and with projected semi-inclusive EIC data (darker bands) [2]. For this analysis only data with $Q^{2}>2.5 \mathrm{GeV}^{2}$ were used, corresponding to $x \geq 0.001$. Center: Same for $\Delta \bar{s}$. Right: Same for the gluon helicity parton distribution, in an analysis by DSSV [10] without (light band) and with projected EIC inclusive data (darker band)

tional contributions are needed from the orbital angular momentum of partons in the nucleon.

An EIC will allow determination of the light-flavor helicity distributions $\Delta u, \Delta d$ and their anti-quark distributions from semi-inclusive scattering, with exquisite precision. By including dedicated studies of kaon production, the strange and anti-strange helicity distributions $(\Delta s$, $\Delta \bar{s}$ ) will also be accessible (see two examples in Fig. 4, left and center panel). Regardless what the combined quark and anti-quark spin contribution to the proton spin turns out to be, comparing the characteristics of the light-sea quark flavor distributions (like $\bar{u}, \bar{d}, s$ and $\bar{s}$ ) and especially their differences will give direct insight in the unique nonperturbative dynamics that generates them. Such studies can be uniquely cross-checked by delineating the flavor structure of the quark and anti-quark helicity contributions with electroweak DIS.

At high $Q^{2}$, the DIS process also gets significant contributions via exchange of intermediate $Z$ and $W^{ \pm}$vector bosons, next to the usual exchange of photons. This gives rise to novel structure functions that are sensitive to different combinations of the proton's helicity distributions. For instance, in the case of charged-current interactions through $W^{-}$, the inclusive structure functions has contributions due to $\Delta u, \Delta \bar{d}, \Delta \bar{s}$ and $\Delta c$, where the latter denotes the proton's polarized charm quark distribution. The analysis of these structure functions does not rely on knowledge of fragmentation, which highlights the complementarity of these measurements to the helicity contributions determined through semi-inclusive scattering. Studies show that both neutral-current and charged-current interactions would be observable at the EIC, even with relatively modest integrated luminosities, roughly $10 \mathrm{fb}^{-1}$.

\section{A QCD in Nuclei Facility}

Measurements of the transverse spatial and momentum distributions of gluons in nuclei could also be performed in a variety of nuclei transcending the regions where the onset of collective behaviour of gluons, and/or the onset of saturation, is found. This would open a new QCD frontier where the onset towards a universal form of gluon matter with characteristic collective behaviour is witnessed. In general, the EIC can explore the 3-dimensional sea quark and gluon structure of a fast moving nucleus, and verify if such structure differs from that of a free nucleon.

The nucleus is a QCD "molecule", with a complex structure corresponding to bound states of nucleons. Understanding the formation of nuclei in QCD is an ultimate long-term goal of nuclear science. The nucleus itself is an unprecedented QCD laboratory for discovering the collective behavior of gluonic matter. The HERA discovery of the large rise of the gluons at small $x$ is a corollary of gluon splitting. But, the gluons can also recombine. It is predicted that, at very high gluon densities, the probability for gluons to recombine must counterbalance the probability for gluons to split, leading to saturation of the gluon density. This state of gluon saturation is predicted to have universal properties inside nucleons and other hadrons, as well as inside all nuclei.

A large occupation number of color charges (partons) leads to a classical gluon field. This could very well dominate the small- $x$ wave-function of the nucleus. This is the essence of the McLerran-Venugopalan model [11]. According to this model, the dominant gluon field is given by the solution of the classical Yang-Mills equations, which are the QCD analogue of Maxwell equations of electrodynamics. This was used to construct an unintegrated gluon distribution (gluon TMD) $\varphi\left(x, k_{T}^{2}\right)$ of a large nucleus, see Fig. 5 (left), multiplied by the phase space factor of the gluon's transverse momentum $k_{T}$. Whereas the gluon distribution is anticipated to rise like $1 / k_{T}$ from perturba- 

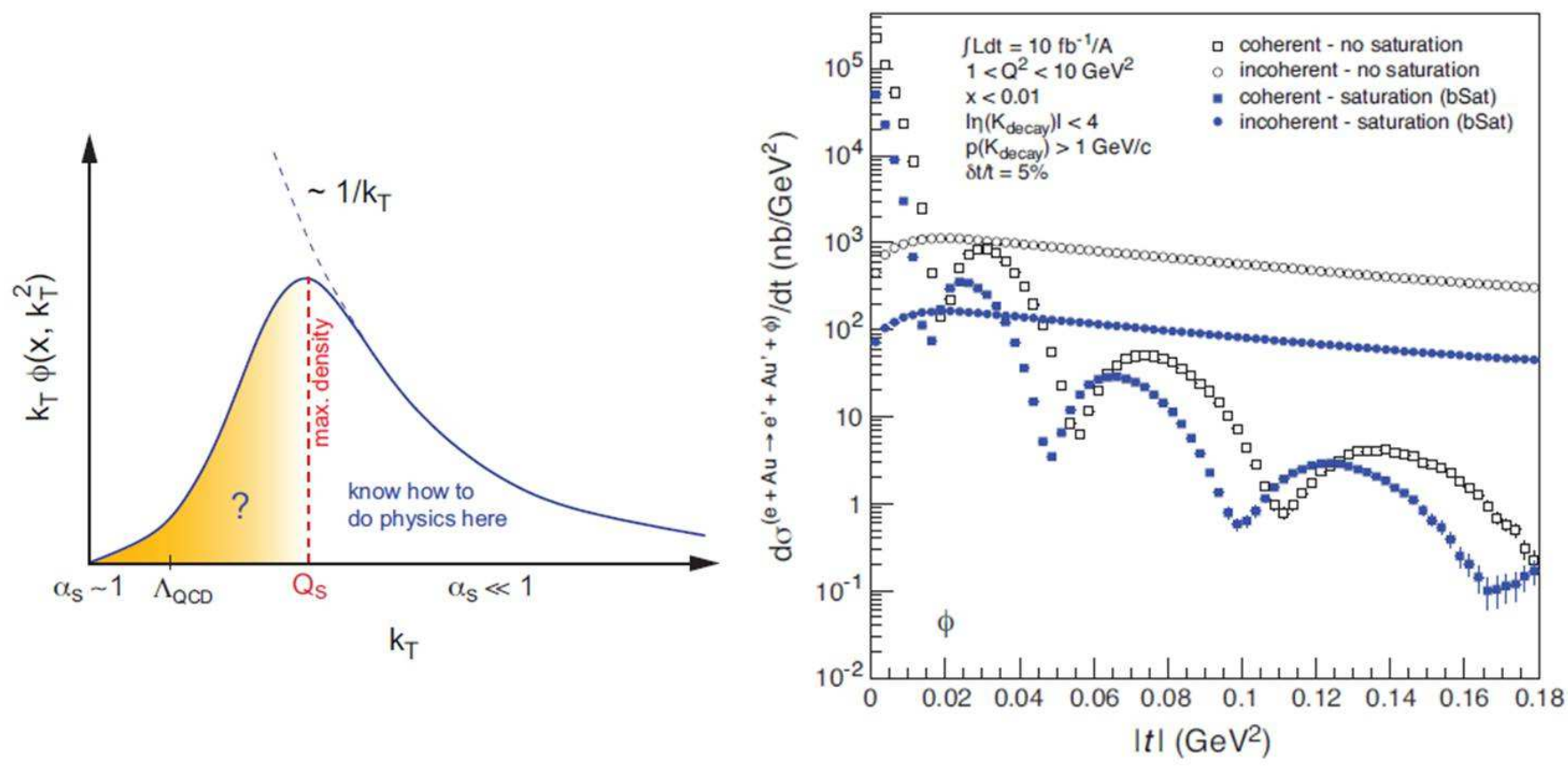

Fig. 5. Left: The unintegrated gluon distribution (gluon TMD) of a large nucleus due to classical gluon fields (solid line). The dashed curve denotes the lowest-order perturbative result and rises like $\sim 1 / k_{T}$. When the gluon density increases, this rise must be tamed. Right: Differential cross section $d \sigma / d t$ distributions for exclusive $\phi$ production in coherent and incoherent events in diffractive e+Au collisions. Predictions from saturation and non-saturation models are shown [2].

tive arguments, one can see a damping beyond the simple multiplication of the phase space factor. The calculated gluon distribution slows down its growth with decreasing $k_{T}$ from a power-law to a logarithm. The majority of gluons in this classical distribution have transverse momentum $k_{T} \sim Q_{s}$. The distribution saturates. This calculation nicely shows the correlation of saturation physics with phenomena accessible in TMD measurements.

With its wide kinematic reach, combined with the capability to probe a variety of nuclei in DIS, semi-inclusive DIS, diffractive and deep exclusive scattering measurements, the EIC can indeed explore the internal 3-dimension sea quark and gluon structure of a fast-moving nucleus. For example, we do not know how gluons are distributed in space - do they follow the confinement radius predetermined by quarks, or whether they contribute to nuclear structure. The momentum distributions of gluons in nuclei is similarly elusive. Due to a near-complete lack of experimental constraints, there is not much knowledge about the possible modification of gluon distributions in nuclei. The gluons could follow the nuclear modifications noted for the quark momentum distributions, known as the nuclear EMC effect, or not. Similar, in the region of smaller $x$ it remains unclear to what extent the gluons are prone to the shadowing effects noted in ratios of nuclear structure functions.

By colliding electrons with heavy nuclei, the EIC will provide access to an uncharted regime of all nuclear matter, where gluons are abundant and dominate its behaviour. With higher gluon densities, the gluons are anticipated to act collectively, rather than independently, and ulti- mately reach a state of saturation, as predicted to be universal in all nucleons and nuclei. Heavy ion beams at the EIC could provide precocious access to the collective behaviour of gluons underlying this phenomenon. The virtual photon exchanged in the electron scattering process probes matter coherently over a characteristic length proportional to $1 / x$, which can exceed the diameter of a Lorentz-contracted nucleus. This implies that the gluons within any nucleon in the nucleus could notice the gluons in another nucleon. This acts as an amplification effect on the gluon density, and is expected to enhance the probability for gluon recombination. Might the effects of gluon recombinations be observed in $e A$ collisions, it would directly point to the collective behaviour of gluons.

If the observation of collective behaviour of gluons would be observed in various nuclei, it would point to the universal property of this effect. Note that the observation of the onset of such gluon recombination effects could very well occur at energies much below those where the gluon splitting and gluon recombination mechanisms are comparable, the latter defining the saturation scale. The onset of saturation itself can lead to dramatic predicted effects in comparisons of $e p$ and $e A$ collisions at an EIC. For example, in some cases the probability for a nucleus to remain intact in a high-energy diffractive process more than doubles in the $e A$ case as compared to the typical $10-15 \%$ probability for diffractive scattering found in $e p$ at HERA.

Extractions of the spatial distributions of gluons in nuclei could be possible from coherent $\phi$ vector meson production in $e A$ scattering, due to the excellent and hermetic 
forward detector capabilities of recoil nuclei. An example for diffractive $\phi$ production in an electron-gold scattering process is given in Fig. 5 (right). These measurements could be performed in a variety of nuclei transcending the regions where the onset of collective behaviour of gluons, and/or the onset of saturation, is found. This would open a new QCD frontier where the onset towards a universal form of gluon matter with characteristic collective behaviour is witnessed.

\section{Outlook}

Due to its unique capabilities for high-luminosity collisions of polarized electrons with polarized protons, polarized light ions, and heavy nuclei, the EIC will, for the first time, precisely image gluons in nucleons and nuclei. The energy reach, luminosity and detection capabilities of the foreseen EIC will allow unprecedented access to the science frontier enabled by the development of the formalism GPDs and TMDs. Multi-dimensional tomographic images will become possible, over a large range of energy scales, from small $x(\sim 0.001$ or 0.0001 or so) to large $x$ (a few $\times$ 0.1 ), exploring the transition of nucleon structure from a region dominated by gluons and sea quarks, to the region of few valence quarks. This will be possible for a large range of resolution, $Q^{2}$, to further validate the theoretical understanding, and for a mutlitude of physics processes. These attributes will make the EIC the ultimate facility for multi-dimensional imaging of nucleons and nuclei with GPDs and TMDs.

The EIC will further definitively reveal the origin of the nucleon spin and will explore a new QCD frontier of ultra-dense gluon fields, with the potential to discover the role of collective effects in nuclei that are expected to lead to a new form of gluon matter predicted to be common to all nuclei. The existence of such dense gluon fields is expected to influence the images of nucleons and nuclei at the smallest energy scales. Lastly, the EIC's hermetic detection capability of correlated fragments promises to finally shed further light on the microscopic origin of the formation of hadrons from quarks and gluons.

Without such a new accelerator facility the EIC we will not be able to understand how the properties and structure of all forms of nuclear matter emerge from the dynamics encoded in QCD, and what role gluons and sea quarks and their interactions play in protons, neutrons, and nuclei. Unambiguous evidence has emerged over the last decades that these both play unexpected roles for defining the structure of nuclear matter around us - an EIC is required to charter and understand those roles.

The EIC was already designated by the U.S. Nuclear Science Advisory Committee (NSAC) in the 2007 Nuclear Physics Long Range Plan as embodying the vision for reaching the next QCD frontier. In 2013 an NSAC Subcommittee report on Future Scientific Facilities declared an EIC to be absolutely essential in its ability to contribute to the world-leading science in the next decade. In the recent 2015 Nuclear Physics Long Range Plan of the U.S. the need for a powerful new EIC to be able to address fundamental questions about the role of gluons in nucleons and nuclei was recognized, and NSAC formally recommended: "We recommend a high-energy highluminosity polarized Electron Ion Collider as the highest priority for new facility construction following the completion of FRIB."

It is clear that the science interest in an Electron-Ion Collider is strong and rapidly growing, worldwide. The science case is strong. These are exciting times for the EIC - now is the time to define realization.

\section{References}

1. D. Boer, M. Diehl, R.G. Milner, R. Venugopalan, W. Vogelsang, et al., arXiv:1108.1713 (2011).

2. A. Accardi, J.L. Albacete, M. Anselmino, N. Armesto, E.C. Aschenauer, et al., arXiv:1212.1701v3 (2014).

3. E.C. Aschenauer, M.D. Baker, A. Bazilevsky, K. Boyle, S. Belomestnykh, et al., arXiv:1409:1633 (2014).

4. S. Abeyratne, D. Barber, A. Bogacz, P. Brindza, Y. Cai, et al., arXiv:1504.07961 (2015).

5. S. Abeyratne, A. Accardi, S. Ahmed, D. Barber, J. Bisognano, et al., arXiv:1209.0757 (2012).

6. J. Dudek, R. Ent, R. Essig, K. Kumar, R. McKeown, et al., Eur. Phys. J. A48 (2012) 187.

7. A. Sandacz, Int. J. Mod. Phys. Conf. Ser. 37 (2015) 1560063 .

8. A. Signori, A. Bacchetta, M. Radici, and G. Schnell, JHEP 11 (2013) 194; Int. J. Mod. Phys. Conf. Ser. 37 (2015) 1560024

9. M. Anselmino, et al, J. Phys. Conf. Ser. 295 (2011) 012062; Eur. Phys. J. A47 (2011) 35.

10. D. de Florian, R. Sassot, M. Stratmann, and W. Vogelsang, Phys. Rev. D 80 (2009) 034030.

11. L.D. McLerran and R. Venugopalan, Phys. Rev. D 49 (1994) 2233. 